\title{
Prospective Determination of Orbital Perfusion Dominance before Intra-Arterial Chemotherapy for Retinoblastoma Using Time-of-Flight Magnetic Resonance Angiography
}

\author{
Eric J. Monroe ${ }^{1}$ Jeffrey P. Otjen ${ }^{2}$ Jason N. Wright ${ }^{2}$ Francisco A. Perez ${ }^{2}$ \\ Jeffrey Forris Beecham Chick ${ }^{3}$ Danial K. Hallam ${ }^{4}$ Mark R. Ferguson ${ }^{2}$
}

${ }^{1}$ Department of Radiology, Section of Interventional Radiology, American Family Children's Hospital and University of Wisconsin, Madison, Wisconsin, United States

${ }^{2}$ Department of Radiology, Section of Pediatric Radiology; Seattle Children's Hospital and University of Washington, Seattle,

Washington, United States

${ }^{3}$ Department of Radiology, Section of Interventional Radiology; Seattle Children's Hospital and University of Washington, Seattle, Washington, United States

${ }^{4}$ Department of Radiology, Section of Neurointerventional Radiology, Seattle Children's Hospital and University of Washington, Seattle, Washington, United States

J Clin Interv Radiol ISVIR 2023;7:64-68.
Address for correspondence Eric J. Monroe, MD, Department of Radiology, University of Wisconsin-Madison, 600 Highland Ave, Madison, WI 53792, United States (e-mail: eric.j.monroe.md@gmail.com).

\author{
Abstract \\ Keywords \\ - retinoblastoma \\ - oncology \\ - intra-arterial \\ chemotherapy \\ - angiography \\ - magnetic resonance
}

Intra-arterial chemotherapy (IAC) represents a mainstay in the treatment of retinoblastoma. In a minority of cases, the external carotid artery (ECA) serves as the dominant supply to the central retinal artery and is associated with prolonged fluoroscopy times and higher intraprocedural radiation doses. The aim of this study was to evaluate the utility of time-of-flight (TOF) magnetic resonance angiography (MRA) for prospective determination of internal (ICA) versus ECA dominance for procedural planning. Between April 2017 and December 2020 (44 months), staging MR prior to IAC for retinoblastoma included variant spatial saturation band position TOF angiography. Exams were then retrospectively reviewed for concordance of ICA versus ECA dominance between the two modalities. Eight consecutive patients were included in the study. Mean patient age at time of diagnosis was $20.3 \pm 10.7$ months (range: $2.7-33.2$ months). Ten affected eyes were included ( 2 cases of bilateral disease), with stage $D$ disease in eight eyes and stage $B$ disease in two eyes. MRA techniques demonstrated antegrade ophthalmic artery (OA) flow in 9/10 (90\%) of affected eyes. Subsequent catheter angiography confirmed ICA dominant supply in 9/9 (100\%). For a single affected eye $(10 \%)$, the OA was demonstrated as orthotopic by T2 flow void, article published online March 4, 2022
DOI https://doi.org/ $10.1055 / \mathrm{s}-0042-1743498$ ISSN 2457-0214.
(C) 2022. Indian Society of Vascular and Interventional Radiology. All rights reserved.

This is an open access article published by Thieme under the terms of the Creative Commons Attribution-NonDerivative-NonCommercial-License, permitting copying and reproduction so long as the original work is given appropriate credit. Contents may not be used for commercial purposes, or adapted, remixed, transformed or built upon. (https://creativecommons.org/ licenses/by-nc-nd/4.0/)

Thieme Medical and Scientific Publishers Pvt. Ltd., A-12, 2nd Floor, Sector 2, Noida-201301 UP, India 
nonvisualized on anterior saturation TOF sequences, and faintly visualized on posterior saturation TOF sequences. Aggregate MRA to catheter angiographic concordance was 10/10 (100\%). Variant saturation TOF MRA predicts ICA versus ECA dominant supply to the central retinal artery in retinoblastoma.

\section{Introduction}

Intra-arterial chemotherapy (IAC) represents a mainstay of therapy for retinoblastoma. ${ }^{1}$ Patients with retinoblastoma, particularly those associated with mutation of the RB1 tumor suppressor gene (RB1), are at risk of secondary malignancies and are sensitive to the ionizing effects of radiation. ${ }^{2-4}$ In a majority of patients, blood supply to the central retinal artery arrives via the internal carotid artery (ICA). In a minority of cases, however, the external carotid artery (ECA) serves as the dominant supply to the central retinal artery most commonly via the middle meningeal artery to the lacrimal artery. ${ }^{1,5}$ While patients with external carotid arterial supply may still be effectively treated by IAC, ${ }^{6}$ this vascular feature has been associated with prolonged fluoroscopy times and elevated procedural radiation exposure. $^{7-10}$ This report describes early experience using variant saturation time-of-flight (TOF) magnetic resonance angiography (MRA) to predict ICA versus ECA dominant supply to the central retinal artery for the purposes of procedural planning.

\section{Technique}

Between April 2017 and December 2020 (44 months), staging MR prior to IAC for retinoblastoma included variant spatial saturation band position TOF angiography. During MRA, two separate noncontrast TOF acquisitions were included in the protocol in addition to routine anatomic sequences. These were coronal acquisitions with coverage including the entire orbit and the bilateral intracranial carotid arteries. The first acquisition included an anterior spatial saturation band to include the bilateral angular arteries and reduce anterior flow-related enhancement. The second acquisition included a spatial saturation band posterior to the orbit to include the bilateral intracranial carotid arteries and nullify posterior flow-related enhancement. All angiographic sequences were standard three-dimensional TOF sequences (field of view (FOV): $200 \times 180$, matrix: $384 \times 365$, repetition time (TR): 26 milliseconds, time to echo (TE): 3.62 milliseconds, slice thickness (ST): $0.45 \mathrm{~mm}$ ), and acquired on a 3-Tesla Prisma Fit MRI (Siemens Healthcare, Erlangen, Germany). Added scan time of the each TOF sequence was $\sim 4.5$ minutes.

All IAC treatments were performed as previously described ${ }^{10}$ using a Philips FD20 bi-plane angiography suite (aSi detector with CsI scintillator, $40 \times 30 \mathrm{~cm}$ detector) with Allura Clarity image optimization. Following catheterization and imaging of the ICA, roadmap angiography was acquired with attention to ophthalmic artery $(\mathrm{OA})$ patency and presence of antegrade flow. Selective catheterization of the OA was performed followed by selective angiography to confirm antegrade flow to the central retinal artery. In cases of nonvisualized OA from the ICA position, the ECA was selected and imaged in a similar fashion. Following confirmation of ECA supply to the OA and central retinal artery, middle meningeal artery to meningo-ophthalmic arterial catheterization was performed to deliver chemotherapeutics.

All cases were then retrospectively reviewed for concordance of ICA versus ECA dominance between the two modalities. Eight consecutive patients were included in the study. Mean patient age at the time of diagnosis was $20.3 \pm 10.7$ months (range: 2.7-33.2 months). Ten affected eyes were included ( 2 cases of bilateral disease), with stage $D$ disease in eight eyes and stage B disease in two eyes. MRA techniques demonstrated antegrade OA flow in 9/10 (90\%) of affected eyes. Subsequent catheter angiography confirmed ICA dominant supply in $9 / 9$ (100\%). A typical case is depicted in -Fig. 1. For a single affected eye (10\%), the OA was demonstrated as orthotopic by $\mathrm{T} 2$ flow void, nonvisualized on anterior saturation TOF sequences and faintly visualized on posterior saturation TOF sequences. Subsequent catheter angiography confirmed ECA dominant supply (-Fig. 2). Aggregate MRA to catheter angiographic concordance was therefore 10/10 (100\%). Procedural fluoroscopy time and radiation for the single case of ECA supply were 5.6 minutes and $8.5 \mathrm{mGy}$, respectively.

\section{Discussion}

This experience suggests variant saturation TOF MRA predicts ICA versus ECA dominant supply to the central retinal artery in retinoblastoma. Routine incorporation into disease staging MR examination may augment IAC procedural planning, potentially promoting procedural expediency and lowering iatrogenic radiation exposure.

Several studies ${ }^{5,6}$ have emphasized that ECA supply to the $\mathrm{OA}$ is most commonly via middle meningeal artery branches (meningolacrimal or meningo-ophthalmic supply) via the superior orbital fissure, or other branches from the posterior orbit. Given the relative rarity of anterior orbital ECA supply (via angular or dorsal nasal artery branches), a single TOF acquisition with anterior saturation may be sufficient to identify absent anterograde OA flow predictive of possible ECA dominance. However, identification of robust reversed flow in an OA may still have prognostic import for the success of IAC. In such cases, adjuvant techniques such as temporary balloon occlusion of the ECA $^{11}$ may be required. Additional MR angiographic techniques such as phase contrast imaging may also determine relative 

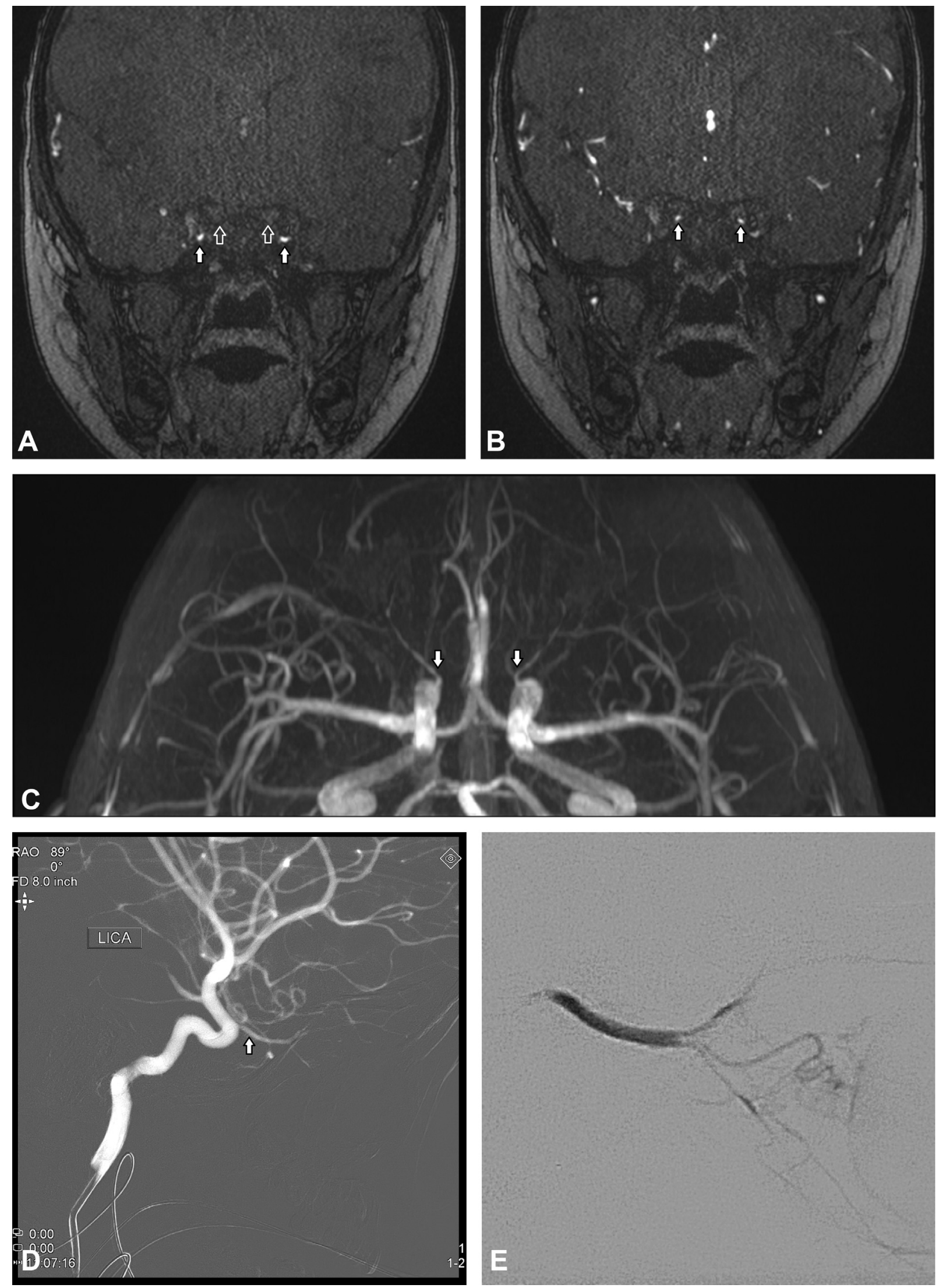

Fig. 1 A 20-month-old male with group D left eye retinoblastoma. (A) Coronal posterior saturation time-of-flight magnetic resonance (MR) image demonstrates high signal within the bilateral superior ophthalmic veins (white arrows) and absence of signal in the expected regions of the bilateral ophthalmic arteries (open arrows). (B) Coronal anterior saturation time-of-flight and (C) axial maximum intensity projection MR images demonstrate high signal within the bilateral ophthalmic arteries (white arrows). (D) Lateral roadmap angiogram of the left internal carotid artery followed by (E) lateral digital subtraction angiogram of the left ophthalmic artery during intra-arterial chemotherapy confirms antegrade flow within the ophthalmic artery. 

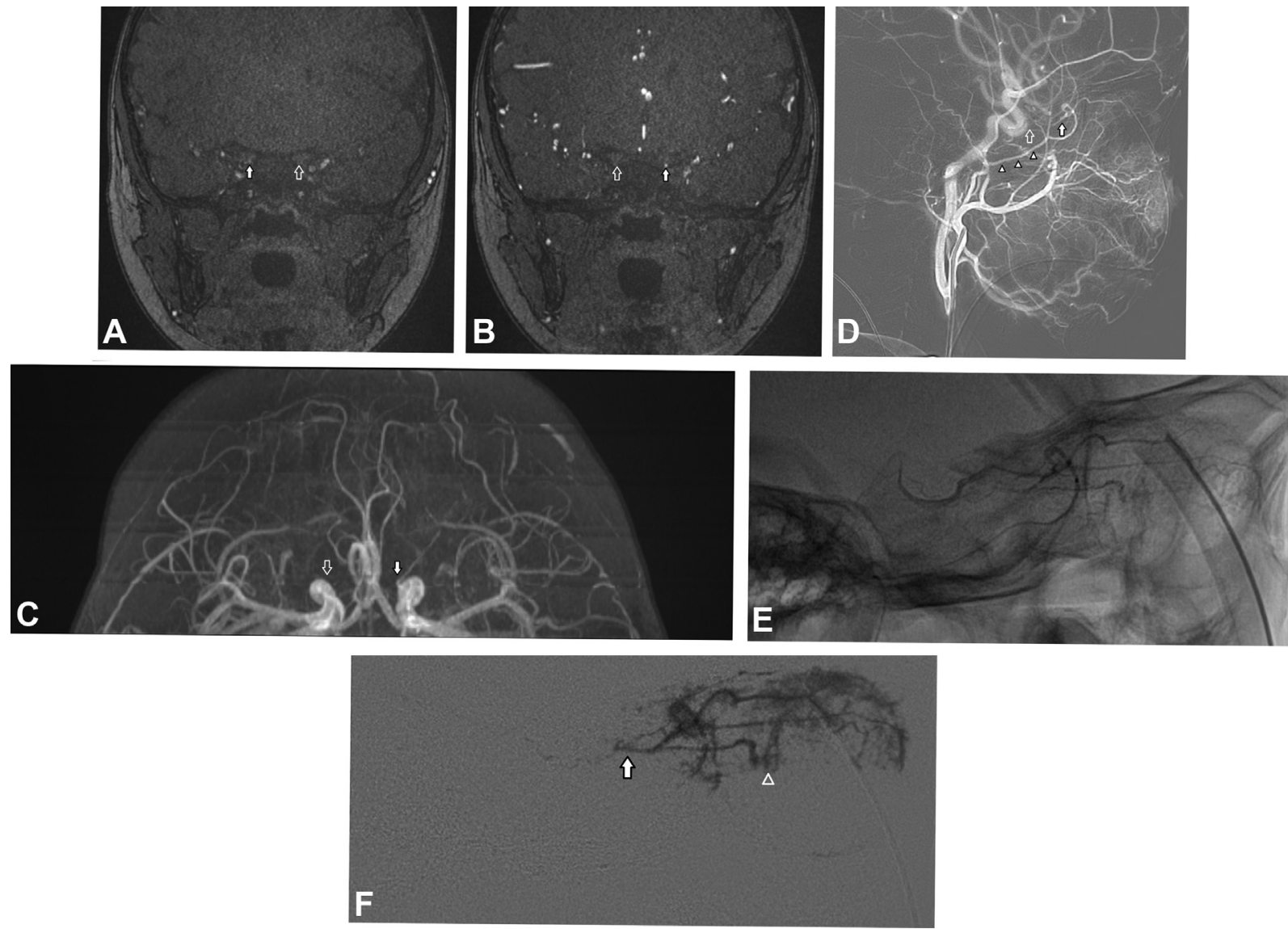

Fig. 2 A 17-month-old male with group D right eye retinoblastoma. (A) Coronal posterior saturation time-of-flight magnetic resonance (MR) image demonstrates faint signal within the right ophthalmic artery (white arrow) and absence of signal in the expected region of left ophthalmic artery (open arrow). (B) Coronal anterior saturation time-of-flight and (C) axial maximum intensity projection MR images demonstrate high signal within the left ophthalmic artery (white arrows) and absence of signal within the expected region of the right ophthalmic artery (open arrows). (D) Lateral roadmap angiogram of the right external carotid artery shows middle meningeal artery (arrowheads) supply to the orbit via meningolacrimal collateralization with opacification of the distal ophthalmic artery (white arrow). Reflux opacification of the right internal carotid artery demonstrates no opacification in the expected location of the proximal ophthalmic artery (open arrow). (E) Unsubtracted and (F) subtracted lateral projection angiograms following catheterization of the meningolacrimal artery confirm supply to the ophthalmic artery (white arrow) with choroidal blush (open arrowhead).

direction of flow in a single acquisition, although the spatial resolution may be insufficient for orbital vessel evaluation. ${ }^{12}$

This study has limitations inherent to small size and retrospective review. In a single affected eye with ECA dominant supply, the $\mathrm{OA}$ was conspicuously nonvisualized on anterior saturation TOF sequences, but only faintly visualized on posterior saturation TOF sequences. Saturation of blood within the middle meningeal artery en route to the orbit may explain this finding. Individual MRA sequence time, total MRA scan time, and total procedural time were not recorded. While procedural fluoroscopy time and radiation for the single case of ECA supply were greatly reduced compared with the institutional performance, ${ }^{10}$ larger series may be needed to solidify the reliability of this technique as well as its impact on procedural planning, total procedure time, and radiation dose reduction.

\section{Conclusion}

Sequential TOF MRA with strategically placed saturation bands accurately predicts ICA versus ECA dominant supply to the central retinal artery in retinoblastoma. Prospective determination of dominant arterial supply may promote procedural expediency and radiation dose reduction during IAC.

\section{Authors' Contribution}

All authors have read and contributed to this manuscript. The authors have no relevant disclosures.

All procedures performed in studies involving human participants were in accordance with the ethical standards of the institutional and/or national research committee and with the 1964 Helsinki declaration and its later amendments or comparable ethical standards.

The study did not receive funding. This study has obtained institutional IRB approval. Informed consent was waived from all individual participants included in the study.

E.J.M., J.P.O., J.N.W., F.A.P. and M.R.F. contributed to the conception. All authors contributed to the design of the research, data acquisition analysis, and data interpretation. E.J.M. drafted the manuscript. All authors critically revised the manuscript, agree to be fully accountable for 
ensuring the integrity and accuracy of the work, and read and approved the final manuscript.

\section{Conflict of Interest}

None declared.

\section{References}

1 Wyse E, Handa JT, Friedman AD, Pearl MS. A review of the literature for intra-arterial chemotherapy used to treat retinoblastoma. Pediatr Radiol 2016;46(09):1223-1233

2 Moll AC, Imhof SM, Schouten-Van Meeteren AY, Kuik DJ, Hofman P, Boers M. Second primary tumors in hereditary retinoblastoma: a register-based study, 1945-1997: is there an age effect on radiation-related risk? Ophthalmology 2001;108(06):1109-1114

3 Draper GJ, Sanders BM, Kingston JE. Second primary neoplasms in patients with retinoblastoma. Br J Cancer 1986;53(05):661-671

4 Kleinerman RA. Radiation-sensitive genetically susceptible pediatric sub-populations. Pediatr Radiol 2009;39(Suppl 1):S27-S31

5 Bracco S, Venturi C, Leonini S, et al. Transorbital anastomotic pathways between the external and internal carotid systems in children affected by intraocular retinoblastoma. Surg Radiol Anat 2016;38(01):79-87

6 Bertelli E, Leonini S, Galimberti D, et al. Hemodynamic and anatomic variations require an adaptable approach during intra-arterial chemotherapy for intraocular retinoblastoma: alternative routes, strategies, and follow-up. AJNR Am J Neuroradiol 2016;37(07):1289-1295

7 Guasti A, Leonini S, Bertelli E, et al. Intra-arterial chemotherapy for retinoblastoma: the dosimetric impact. Neuroradiology 2019; 61(09):1083-1091

8 Area C, Yen CJ, Chevez-Barrios P, et al. Technical and anatomical factors affecting intra-arterial chemotherapy fluoroscopy time and radiation dose for intraocular retinoblastoma. J Neurointerv Surg 2019;11(12):1273-1276

9 Qureshi AM, Davies LK, Patel PA, Rennie A, Robertson F. Determinants of radiation dose in selective ophthalmic artery chemosurgery for retinoblastoma. AJNR Am J Neuroradiol 2019;40(04): 713-717

10 Monroe EJ, Chick JFB, Stacey AW, et al. Radiation dose reduction during intra-arterial chemotherapy for retinoblastoma: a retrospective analysis of 96 consecutive pediatric interventions using five distinct protocols. Pediatr Radiol 2021;51(04):649-657

11 Abruzzo TA, Geller JI, Kimbrough DA, et al. Adjunctive techniques for optimization of ocular hemodynamics in children undergoing ophthalmic artery infusion chemotherapy. J Neurointerv Surg 2014

12 Bitar R, Leung G, Perng R, et al. MR pulse sequences: what every radiologist wants to know but is afraid to ask. Radiographics 2006;26(02):513-537 